\title{
Keeping orthodontic treatment results steady
}

\author{
J. Philippe
}

\begin{abstract}
To maintain treatment effects as long as possible, a functional balance has to be struck, stabilizing the underlying morphologic elements. Form should match function, and function should match form, according to the type of dysmorphia treated.

To this end, certain morphological and behavioral features have to be included in the treatment plan, and achieved by end of treatment, with follow-up after retention, so as to maintain them throughout life.
\end{abstract}

\section{KEYWORDS}

Retention, maintaining treatment effects, finishing, relapse, stability

"As long as it lasts!" (Laetitia Bonaparte)

\section{INTRODUCTION}

Since the publications of Little's long-term treatment results ${ }^{6,7}$, a wave of pessimism has engulfed the orthodontic community. Little reported a very large number of cases managed at the University of Washington, $70 \%$ of which relapsed at 10 years' followup and $90 \%$ at 20 years, whatever the type of malocclusion or treatment. The pessimism was darkened by further studies confirming instability of outcome in studies that were not the practitioner's own report (Nanda ${ }^{8}$.
We later gained a better understanding of what should be called relapse and what is more a matter of the natural maturation of the face. Aging entails a reduction in arcade dimensions which was described as a "centripetal drift"9.

It is also noteworthy that the cases studied by Little had been managed several decades before the study, by American practitioners, inspired by the ideas of the time, which were founded more on "normal values" (Tweed's cephalometric

Address for correspondence:

Julien Philippe

6 rue Chanzy 
norms for the face and the BonwillHawley geometric norms for the arcade) than on an objective of functional balance.

Nowadays, the face is no longer seen as a collection of cephalometric angles, but as an organ. And a living organ. It can no longer be analyzed in two dimensions, but rather in four, the most important of which is the fourth: time; the face is constantly changing, from youth to age, and from the end-of-treatment alignment to elderly dentition.

Retention devices only last for a certain (short) time, before the form of the face and arcades comes under the sway of facial function and tension. This is why, at end of treatment, functional flexibility has to be easy enough and effective enough to maintain the morphological elements on which it is based. However, functional ease conversely depends on favorable morphological conditions. And moreover, the interdependent whole constituted by form and function obviously has to be adapted to the individual and the type of malocclusion treated.

Life, as Bichat put it, is that set of functions that counters death, and there are functions that counter facial aging. According to Talmant, DFO is the specialty that places teeth and jaws in the position in which aging will proceed as well as possible ${ }^{16}$.

Treatment should be inspired by the aim of achieving lasting results as much as of improving function and appearance; this is an integral part of orthodontic treatment, which cannot be designed to be ephemeral.

Concretely, this aim of maintaining results and helping resist aging takes the form of morphological and behavioral measures included in the treatment plan, achieved in implementing treatment, maintained by means of retention, checked after retention, and kept steady lifelong.

\section{TREATMENT PLAN}

For treatment effects to be lasting, the treatment plan should meet certain requirements.

- It should be based on complete diagnosis: if etiology cannot be identified, the cause, which is often functional, will persist, leading to relapse.

- It must be realistic: the most ambitious treatments are also the least stable. Implementing alveolar compensation when surgery is indicated is highly risky.
- Maintaining treatment effects often involves behavioral rehabilitation, which may be long and difficult. Firm consent by a well-informed patient is indispensable.

- Planning should take account of particularities of head shape (small face, oral orifice size, muscle strength, etc.), goodness of match between statural growth and dental age, and the type of facial growth: there is no one "standard" treatment".

- The particularities of the various functions should be looked into 
and taken into account: notably, symmetry of facial expression, respiration and mastication: there is no "one-size-fits-all" rehabilitation.

- According to most authors (Kerosuo $^{5}$, Rollet $\left.{ }^{15}\right)$, early treatment ensures greater stability.

- Deciding to reduce dental material or not, by extraction or coronoplasty, should take account of long-term stability, as should the choice of teeth for extraction. If only maxillary extraction is planned, long-term mandibular growth may induce diastemas between the maxillary incisors.

- Pre-treatment incisor remodeling should not be based on purely esthetic considerations, but also eliminate causes of instability. Disproportion between maxillary and mandibular incisors should be corrected by coronoplasty; marginal crests that may persist on the lingual sides of the maxillary incisors impair mandibular incisor positioning and should be milled down (Philippe ${ }^{11}$.

- Arcade shape should not be dictated, as used to be the case, by geometrical theories, or, as presently, by esthetic considerations without regard for functional balance.

- It is unwise to plan wide arcades in a narrow face.

- The opinion of relevant professionals (ENT, surgeon, physiotherapist, prosthetist) should be sought at the appropriate time.

\section{ACTIVE TREATMENT}

The mistakes in treatment that most often impair stability are the following:

- Lack of rehabilitation: not all dysfunctions and negative behaviors are corrected.

- At end of treatment, maximal intercuspal occlusion corresponds poorly to the centered relation. A discrepancy between these two positions entails risk of temporomandibular joint disorder, dental wear and recurrence of malocclusion due to mandibular withdrawal.

- Symmetry of mastication, lateral sliding and the occlusal plane in frontal view have not been restored.

- Arcade form is considerably altered without corresponding change in facial balance.
- The arcade has been remodeled according to a "standard", and does not match the patient's facial anatomy or physiology.

- Intercuspation is inexact. Notably, the mesiolingual maxillary molar cusps are not centered on the mandibular molars but on a cuspid side, displacing them (see note $\mathrm{n}^{\circ} 2$ and fig. 1).

- Closed bite may be corrected by incisor intrusion or vestibular version (fig. 2), molar extrusion or a combination of the three. Intrusion is the least subject to muscle action, and seems most stable $\left(V_{a r l i k}{ }^{17}\right)$. Correcting closed bite by continuous curved elastic arches in the vertical plane shows high risk of relapse, inducing incisor version with extrusion only of the 


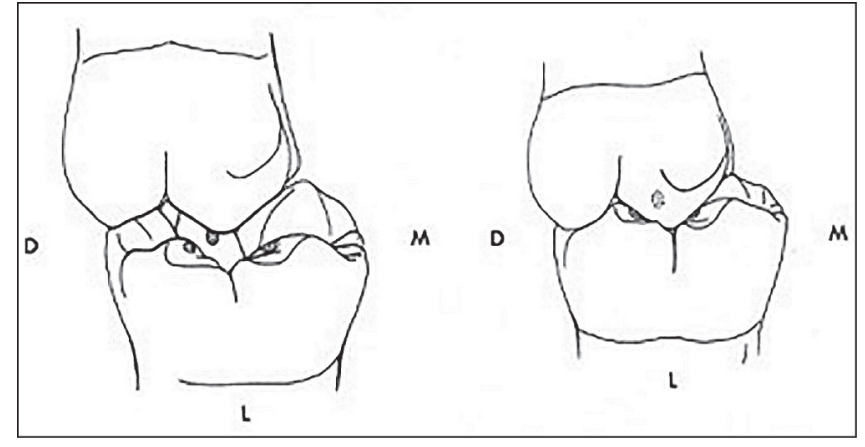

Figure 1

(From Marguelles-Bonnet and Yung, Pratique de I'analyse occlusale et de l'équilibration, Ed. CdP.) Balanced contact (tripodal) of mesiopalatal cusp in opposite fossa. Contact on a single side would induce parasitic forces and tooth displacement.

premolars, which are both factors of relapse.

- Strong rotation should have been slightly overcorrected (see note $\left.n^{\circ} 5\right)$.

- Several overcorrections remain at end of treatment, preventing balanced occlusion; this should be avoided (see note $n^{\circ} 5$ ) .

\section{RETENTION}

Retention aims to prevent the ligament and gum fibers, stretched or compressed by orthodontic displacement, from returning the tooth to its former position.

A wide variety of mechanical devices enable such retention ${ }^{10,11}$, and should:

- be applied immediately: the movement toward relapse begins 2 hours after removing the active device (Reitan, confirmed in 2013 by Franzen $\left.{ }^{4}\right)$;

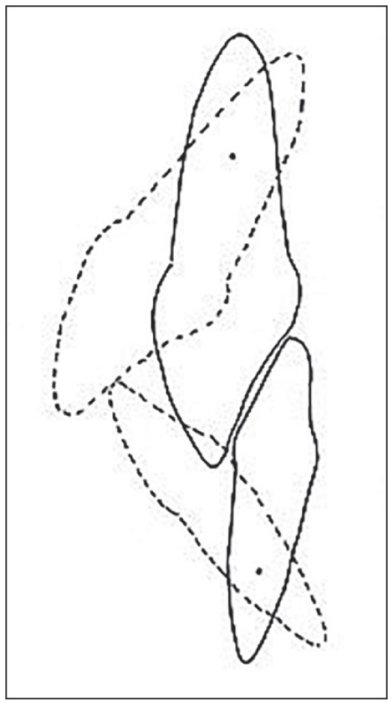

Figure 2

Incisor vestibular version reduces overlap. It is a "pseudo-intrusion," according to Burstone; no forces counter maxillary incisor extrusion.

- Treatment should, also and above all, ensure means adapted to the malocclusion being treated: see below, "Control of stabilizing factors".

- be designed to resist foreseeable displacement precisely: a wire glued from canine to canine will not maintain molar expansion, and a Hawley plate will not maintain canine rotation:

- be fixed: removable devices are too accident-prone, which may ruin the results of the best treatment. If a removable device is absolutely required, the patient should be given two of them, to alternate ${ }^{10}$; 
- remain in place until the desmodontal and gingival fibers cease to exert traction or pressure: i.e., until they get reorganized, in view of alveolar bone apposition/resorption. This takes at least 2 or 3 years, and probably longer in adults and for rotations.

It should be borne in mind that many devices that are used provide only partial retention, be it in space (wire glued from canine to canine) or time (device abandoned before desmodontal fiber reorganization is complete), or intentionally (no devic-

\section{CONTROL OF STABILIZING FACTORS}

Control seeks to check, pursue and complete what has been done in drawing up and implementing the treatment plan and by retention, with a view to maintaining outcome.

As we are talking about long-term results, it makes more sense to put our trust in morphologic and behavioral factors than in mechanical systems that often go wrong.

Most factors are achieved by active treatment and can be checked only at this stage; but others, such as behavioral change, can be pursued during retention, while yet others, such as occlusal balance, can be achieved with greater precision only once the apparatuses are removed.

The factors vary somewhat according to the type of dysmorphism.

- Maintaining endognathic and endoalveolar treatment effects presupposes: es, except positioners, maintain interarcade relations). Partial retention means stability that is limited in space or time. In practice, the extent and duration of retention represent a compromise between desired stability, discomfort and the desire to finish treatment as soon as possible. (Here would be a good place for a section on applied psychology, applied to the practitioner, the patient and the family; but we have neither the space nor the expertise.)

Research has been done to find a process that would activate desmodontal fiber reorganization.

- day- and night-time nasal respiration (role of ENT);

- tongue remaining within the arcades at all times (swallowing, enunciating, sleeping, etc.);

- active mastication (chewing-gum);

- mastication balanced between left and right, in principle by alternating movements; this may require adapting the lateral cusps (Planas);

- arcade width matching the shape of the face;

- well-matched mandibular and maxillary arcade widths;

- no molar vestibular version after expansion;

- relaxed facial envelope activity: early onset of nasolabial folds is a bad sign.

The first 4 points, and the last (which is progressive), should be checked periodically throughout life. 
- Maintaining treatment effects in tooth-arcade dysharmony (e.g., DMD) presupposes:

- frequent tongue pressure against the mandibular incisors, with arcades closed (see note $n^{\circ} 1$ );

- good neutralization of facial envelope muscles (attention to tics and facial expression);

- harmony between mandibular and maxillary tooth dimensions (Bolton index), which should have been rendered proportional by coronoplasty at start of treatment;

- low anterior tooth overlap (see note $n^{\circ} 1$ and fig. 3);

- precise intercuspation, especially molar (see note $n^{\circ} 2$ and fig. 1);

- perfect occlusal balance, in maximum intercuspation and function (see note $n^{\circ} 3$ );

- root parallelism, in case of extraction;

- mandibular incisor beveling, in case of coronoplasty (fig. 4);

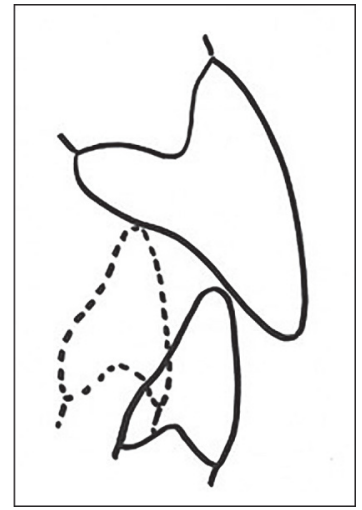

Figure 3

With increasing overlap, mandibular incisors and canines tilt lingually, inducing incisor malpositioning and decreased intercanine distance in the mandible.

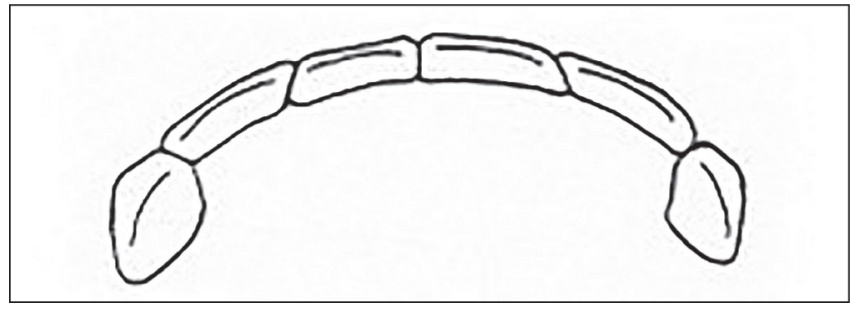

Figure 4

(From Barrer, Revue d'ODF 1975;9:363-72.) Mandibular incisor beveling to prevent recurrence of rotation.

- long mechanical retention of rotation (wire glued to 2 adjacent teeth).

All these points should be periodically checked throughout life.

- Maintenance of closed bite treatment presupposes:

- tip-to-tip incisor contact in forward and lateral sliding (fig. 5), the frequency of which can be seen from wear facets appearing on the incisor edges and the reciprocal adaptation of maxillary and mandibular incisors when positioned tip to tip: tooth extrusion is stopped only by an opposing force ${ }^{11,12}$;

- in the absence of such contact, after checking that it is not being prevented by occlusal interference, a little cingular block should be maintained on the anterior incisors throughout life (fig. 6);

- growth in strong anterior rotation is another indication for fitting a cingular block;

- correction of closed bite by incisor intrusion is more stable than by incisor vestibular version (fig. 2) or molar extrusion (Varlik ${ }^{17}$ ).

- NB: slight lingual interposition while pronouncing sibilants can ensure retention of closed bite. 


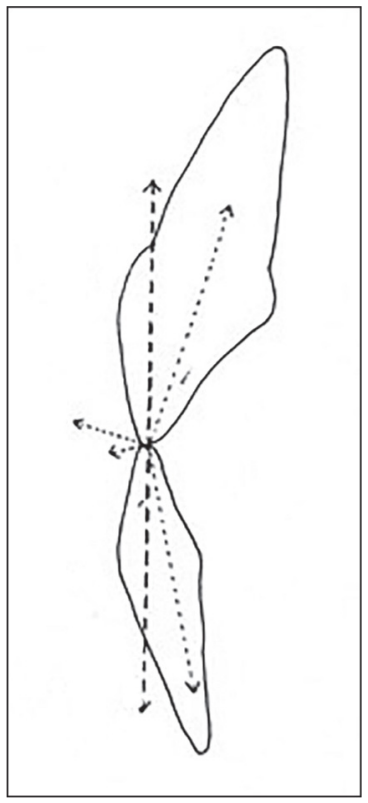

Figure 5

Tip-to-tip incisor contact exerts force opposing extrusion.

- NB: A small interincisor angle (incisor vestibular version) reduces overlap (fig. 2). Occlusal contact then tends to shift the maxillary incisors increasingly toward vestibular version, while opposing no force against their natural extrusion. If the orbicular is not active, the incisors will tilt and continue to extrude, without increasing overlap. If the orbicular is active, they will move backward in straightening, and overlap will increase, with recurrence of closed bite.

The first two points progress naturally, and need periodic checking throughout life.

- Maintaining class II, div. 1 malocclusion treatment effects presupposes:

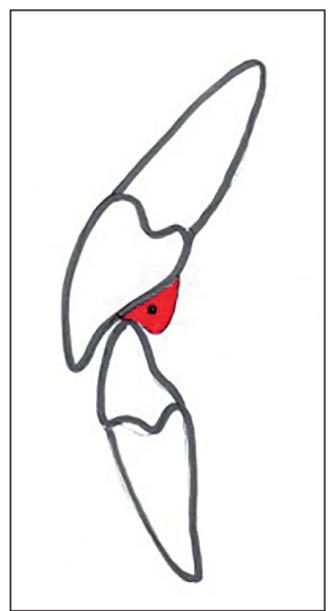

Figure 6

Cingular block, positioned during retention wire gluing. This little occlusal face opposes incisor extrusion in the absence of tip-to-tip contact.

- achieving all the above endognathic and endoalveolar points;

- good (almost coinciding) relation between occlusion in maximal intercuspation and centered relation (fig. 7): if this is satisfactory, malocclusion treatment is generally maintained by the final phase of mandibular growth, which continues longer than maxillary growth (see note $\mathrm{n}^{\circ} 4$ );

- repeated contact between upper and lower lips, reinforced if need be by appropriate exercises;

- maintaining the vestibulo-distal rotation of the maxillary molars, as classically in non-extractive treatment of class II (very important);

- precise, deep intercuspation (see note $\mathrm{n}^{\circ} 2$ and fig. 1);

- mastication exercises (chewinggum); 


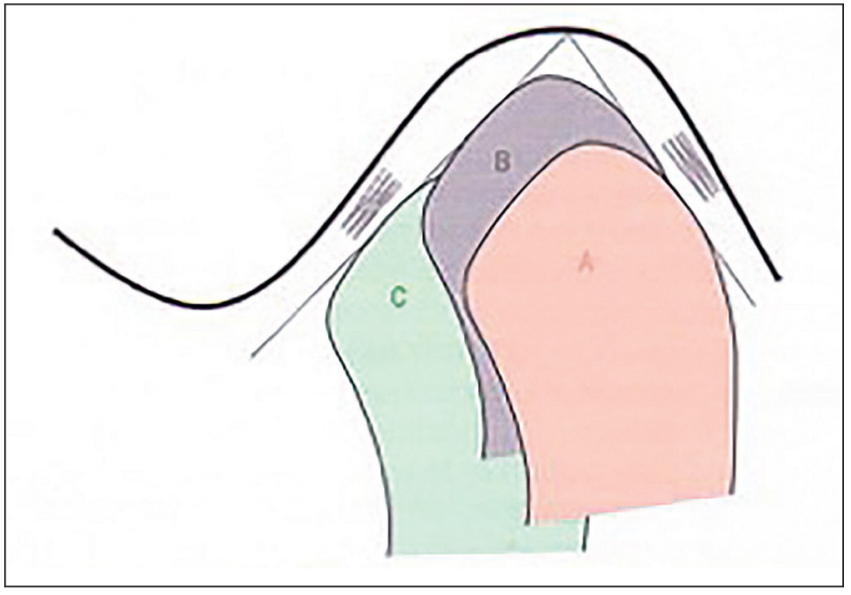

Figure 7

(From Legall MG and Lauret JF, Occlusion et fonction, Ed. CdP.) In B, centered relation of condyles in glenoid cavity.

- good occlusion plane orientation (raised forward, lowered backward).

All these points need periodic checking throughout life.

\section{ALL LIFE LONG}

The functional balance achieved by the above measures has to be checked and maintained all life long.

- Morphological and behavioral checks (stability of results, occlusal balance, absence of harmful behavior, and functional symmetry) should be made regularly, throughout life, just as descaling is necessary all life long. The cingular blocks are subject to wear by mastication, and need periodic renewing.

- Daily exercises may have been prescribed, such as lingual pressure
- Maintaining class III malocclusion treatment effects presupposes:

- including in the treatment plan the disastrous effect of the final mandibular growth spurt;

- day- and night-time nasal respiration (role of ENT);

- secure vertical dimension, increase in which induces posterior rotation of the mandible: no extraction, no milling, no mastication exercises (no chewing-gum);

- good occlusion plane orientation (lowered forward, raised backward) (Raymond ${ }^{14}$ ) ;

- if the mandibular molars have been extruded (to increase the vertical dimension and incline the occlusion plane), the extrusion needs supporting, keeping the wisdom teeth, which have plenty room in a large mandible;

- precise, deep intercuspation (see note $n^{\circ}$ 2) with increased incisor overlap.

on the incisors: these form part of daily oral hygiene.

- Glued cast splint. There is a device that prevents tooth displacement under desmodontal fiber traction and ensures arcade stability even when the above-listed maintenance conditions are not all met: a cast metal (i.e., rigid) glued splint.

This device is suited only to adult dentition, but usually meets objectives; however, it fails to maintain anteroposterior arcade relations, and entails a major risk: partial detachment. we have encountered one such 
device, glued to the whole maxillary arcade, but unglued from the central incisor. Severe decay developed that could not be treated with the splint in place, and the splint could not be removed due to the condition of the posterior periodontium. This just goes to show how carefully these devices need to be designed, produced, glued and balanced.

If occlusal contact, whether centered or functional, is applied not on the splint but on the teeth, the splint tends to detach. Contact should therefore be on the splint directly, complicating design.

This splint provides long-term retention, without taking account of physiological factors which, without the splint, would lead to recurrence. There is a fear (without evidence, to our knowledge) that the persistence of these factors may be harmful for the periodontium or temporomandibular joint.

\section{CONCLUSION}

In 2000, we published a little book on recurrence and retention ${ }^{11}$, with two final pages of conclusions, beginning as follows: "Stability of results should be sought, first and above all, by the treatment and then by retention. There can be no stability if diagnosis is incomplete, if the treatment plan is not drawn up with stability in mind, if the morphological result is not adapted to functional forces, and if intercuspation is not balanced. Retention, as much as treatment, has to be specifically adapted to each case, and should last as long as it must."

This advice was followed by M. Saadia's story of "Six blind men and the elephant".

Neither the advice nor the story seem outdated.

\section{NOTE $N^{\circ}$ 1. Incisor malpositioning}

One feature of facial aging is an increase in lip pressure on the incisors and canines, starting at the end of adolescence (centripetal drift).
Young people's lips are usually fleshy, soft and seemingly flourishing. With adulthood, they become thinner and exert stronger pressure on the anterior arches of both arcades. The upper lip lengthens, as the tip of the nose drops (Behrents ${ }^{2}$ ), and pressure moves downward. This increased centripetal labial pressure is not compensated by an increase in centrifugal lingual pressure. On the contrary, suprahyoid muscle strap ptosis, which comes into play before the age of 40 , and reduced submaxillary gland volume drive the tongue backward from its usual position. Consequently, the incisors withdraw, malpositioning appears and intercanine distance diminishes in both arcades. If overlap increases due to lingual version of the maxillary incisors, or for any other reason, there is less and less room for the mandibular teeth and intercanine distance diminishes more in the mandible than in the maxilla (fig. 3). 
These are average tendencies, with wide individual variation. We have encountered subjects whose incisor alignment never changed over the years; but we have also treated women at the age of 40 whose incisors were perfectly well aligned in their 20s.

There are 4 means of countering this trend.

1) Restoring labio-lingual muscle balance by a very easy and discreet exercise: press the tongue strongly against the incisors, several times a day, with the arcades closed tight. So as not to get forgotten, the exercise can be combined with some repetitive physiological action.

2) Trusting dental arcade resistance: in architecture, an arch can bear considerable weight. This presupposes perfect alignment of the points of contact of the 6 anterior teeth of both arcades. By precaution, mandibular arch resistance can be strengthened with a 33-43 wire, removed and replaced every 3 years; a round $0.7-\mathrm{mm}$ wire is preferable to a twisted wire.

3) Avoiding increased overlap, which leaves less room for the mandibular incisors (fig. 3). Excessive overlap prevents good alignment of mandibular incisors that are not microdontal. In practice, this may mean fitting a cingular block (fig. 6).

4) Regularly applying the individual positioner at end of treatment.

These recommendations should be followed life-long.

\section{NOTE $\mathbf{N}^{\circ}$ 2. Intercuspation in maximum occlusion}

Intercuspation interrelates the arcades and is an important factor in maintaining occlusion relations.

A principal factor is the contact between the maxillary molar mesiopalatal tip and the central fossa of the mandibular molars (fig. 1). Contact can be assessed from the trace left by articulating paper or a silk ribbon; otherwise, there is a problem, as cusp tip position cannot be seen within the mouth. Is it too high, too forward, too backward? Only molding (which can be limited to the occlusal sides) can judge. Also, with an edgewise arch (a reciprocal anchoring system), it is very hard to displace the palatal cusps of both maxillary molars into the fossa of both mandibular molars. This is why intercuspation often fails to be achieved, because it cannot be seen - yet occlusion remains unstable !

Canine occlusal contact, ensuring lateral blocking, should also be checked, balanced and rendered symmetrical by milling or introducing composite.

The best device for finishing and intercuspation is a positioner in rubber (the only really elastic material) following an individual set-up on an adapted articulator. If the impression is taken before removing the brackets and the device is positioned just after, wearing it continuously for one weekend is highly effective. It may thereafter be worn part-time or replaced by a more convenient, less visible device. There is no such thing as a "standard" positioner. 


\section{NOTE $N^{\circ}$ 3. Occlusal balance}

This is a complex issue. Here, we shall just look at a few points:

1) Mandible position in maximum intercuspation should coincide with that in an anteroposteriorly and, more strictly, transversally centered condyle relation (fig. 7). Occlusal prematurity deviating the mandible in maximum intercuspation does not correspond to a centered relation and should be carefully screened for and eliminated by displacing or milling the culprit tooth.

2) At end of treatment, occlusal contacts should be numerous and well-distributed over the arcades. The same holds true for each individual tooth: a molar with occlusal contact limited to one mesial or distal crest (fig. 8) is obviously unbalanced, and will move ${ }^{13}$. This needs correcting by milling, if contact is premature, or, more frequently, by introducing composite on the nonfunctional crest (fig. 9).

The "classic authors" have, with a wealth of detail, described all the orientations, relations and positions that teeth should have for perfect occlusal balance. Their theories have recently been well summarized by Fourquet ${ }^{3}$. All deserve respect, and all are exact so long as tooth shape and function match theory; otherwise, they are all wrong.

3) Forward slide should be guided by the incisors, enabling tip-totip incisor contact. Lateral slide should be symmetrical (Planas), guided by the vestibular cusps of the teeth on the working side,

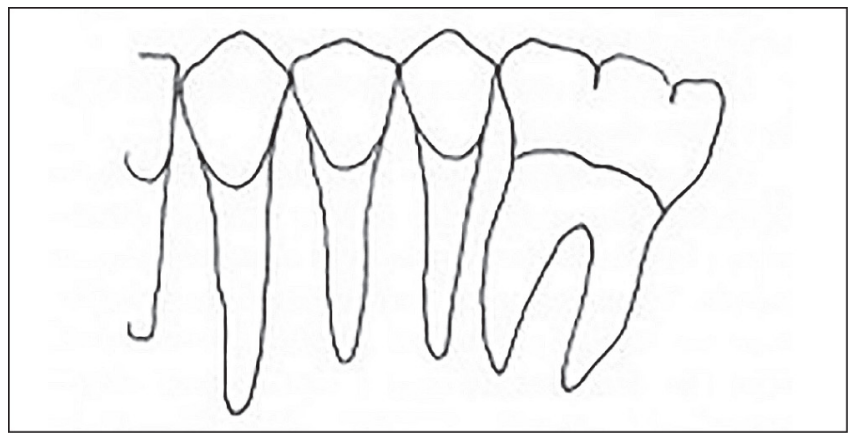

Figure 8

Molars in distal version have occlusal contact only on the mesial crest, inducing mesial version.

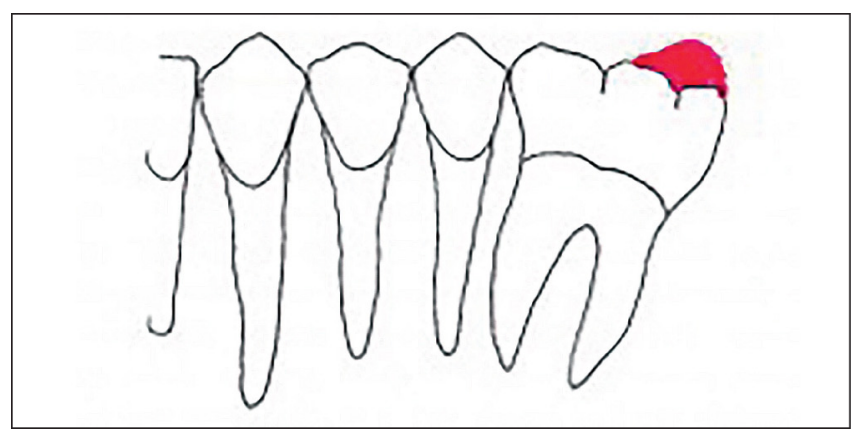

Figure 9

Molars in distal version can be rebalanced by introducing composite to restore distal occlusion contact.

and passing through unilateral tip-to-tip incisor contact; Symmetric functional movement, especially in facial expression and mastication, should be carefully checked.

4) Although occlusal balance should, in theory, be ensured by treatment and finishing (positioner), posttreatment rebalancing of both static and dynamic occlusion by milling and composite greatly helps reduce unwanted subsequent displacement. 


\section{NOTE $\mathrm{N}^{\circ} 4$. Correction of class II, div. 1 malocclusion}

The anatomic effects of correcting class II, div. 1 malocclusion are several.

1) The maxilla and nasal cavities are affected by treatment (maxillary disjunction) and by nasal breathing, jointly achieved by ENT and orthodontist.

2) The alveolar-dental structures of both arcades are displaced in counter-directions by treatment, whether functional or mechanical. These displacements tend to relapse, and can only be maintained by a retention device including both arcades (positioner) and/or precise and deep intercuspation, reinforced by powerful mastication (Antonarakis ${ }^{1}$ ) (chewing-gum).

3) Mandibular growth or, more exactly, the posteroanterior difference between mandibular and maxillary growth, is not subject to relapse. It is inconstant, uncontrolled by treatment, and may show late onset. It usually has positive impact, compensating alveolardental relapse and inducing mental advancement. These effects may be reduced by posterior rotation of the mandible, possibly due to treatment. Whatever the treatment, maximum intercuspation occlusion should more or less coincide with a centered condylar relation, at end of treatment or later (end of retention). This relation should be especially carefully checked if treatment involved temporarily positioning the condyle forward of the centered position (use of activator, connector, etc.), counting on growth to recenter the condyle.

4) The greater the inclination of the occlusion plane, downward in front and backward behind, the more the occlusal forces push the maxillary arcade forward and the mandibular arcade back. At the very least, treatment should not aggravate this inclination.

\section{NOTE $N^{\circ} 5$. Overcorrection}

Reitan recommended displacing the tooth beyond the target position, to stretch the ligament fibers as much as possible, then bringing the tooth back on target. Stretched ligament fibers are less liable to induce recurrence. This overcorrection during treatment is particularly recommended for rotation, which is highly subject to recurrence under ligament traction.

Many classical authors recommended ending treatment with the teeth in overcorrection, to preventively compensate relapse: it was recommended to overcorrect rotation, leave molars in distal version, flatten the Spee curve and reduce incisor overlap, counting on relapse to sort things out. In our view, causing interference, creating imbalance and destroying the incisor guide is no way to enhance stability: balanced occlusion is more stable than unbalanced occlusion, and clearing the way for relapse is playing with fire.

Conflict of interest: The author declares no conflicts of interest. 


\section{REFERENCES}

1. Antonarakis GS, Kjellberg $\mathrm{H}$, Kiliaridis $\mathrm{S}$. Bite force and its association with stability following ClassII/1 functional appliance treatment. Eur J Orthod 2013; 35:434-441.

2. Behrents RG. Growth in the aging cranio-facial skeleton. University of Michigan, 1985.

3. Fourquet L, Göttle M, Bounoure G. Finitions, stabilité et harmonie. Orthod $\mathrm{Fr}$ 2014;85:93-125.

4. Franzen TJ, Brudvik P, Vandevska-Radunovic V. Periodontal tissue reaction during orthodontic relapse in rat molars. Eur J Orthod 2013;35:152-159.

5. Kerosuo H, Heikinheimo K, Nyström M, Väkiparta M. Outcome and long-term stability of an early orthodontic treatment strategy in public health care. Eur J Orthod 2013;35:183-189.

6. Little RM, WallenTR, Riedel RA. Stability and relapse of mandibular anterior alignmentfirst premolar extraction cases treated by Edgewise orthodontics. Am J Orthod 1981;80:349-365.

7. Little RM, Riedel RA, Artun J. An evaluation of changes in mandibular anterior alignment from 10 to 20 years postretention. Am J Orthod Dentofacial Orthop 1988;93:423-428.

8. Nanda RS, Burstone C. Retention and stability in orthodontics. Philadelphia : WB Saunders, 1993.

9. Philippe J. Récidive orthodontique et dérive centripète. Rev Orthop Dento Faciale 1989;23:317-328.

10. Philippe J. La contention. Rev Orthop Dento Faciale 1993;27:317-328.

11. Philippe J. La récidive et la contention post-orthodontique. Paris : Ed. SID, 2000, 151 p.

12. Philippe J, Lejoyeux E. La supraclusion incisive : sa pathogénie et la stabilité de traitement. L'Orthodontie Bioprogressive 2009:9-15.

13. Philippe J. Les multiples causes de la récidive. Orthod Fr 2005;76:183-186.

14. Raymond JL. Traitement orthopédique des malocclusions de Classe III. Paris, Empressa, 2002, $158 \mathrm{p}$.

15. Rollet D. L'éducation fonctionnelle : prévention et interception des malpositions dento-maxillo-faciales, amélioration des problèmes fonctionnels de l'adulte. Orthod Bioprogressive 2014;22:11-22.

16. Talmant J. Journées de la SBR. Marseille. Sept. 2012.

17. Varlik SK, Alpakan OO, Turkoz C. Deepbite correction with incisor intrusion in adults: A long-term cephalometric study. Am J Orthod Dentofacial Orthop 2013;144:414-419. 